\title{
Does the RIFLE Classification Improve Prognostic Value of the APACHE II Score in Critically Ill Patients?
}

\author{
Kátia M. Wahrhaftig, Luis C. L. Correia, Denise Matias, and Carlos A. M. De Souza \\ Department of Postgraduate Medicine and Human Health, Bahia School of Medicine and Public Health (EBMSP), \\ Avenida D. João VI 275, PAV.II, $2^{\circ}$ Andar, Sala 07, Brotas, 40.290-000 Salvador, BA, Brazil \\ Correspondence should be addressed to Kátia M. Wahrhaftig; katitamw@hotmail.com
}

Received 5 May 2013; Accepted 17 July 2013

Academic Editor: Jaime Uribarri

Copyright (C) 2013 Kátia M. Wahrhaftig et al. This is an open access article distributed under the Creative Commons Attribution License, which permits unrestricted use, distribution, and reproduction in any medium, provided the original work is properly cited.

\begin{abstract}
Introduction. The RIFLE classification defines three severity criteria for acute kidney injury (AKI): risk, injury, and failure. It was associated with mortality according to the gradation of AKI severity. However, it is not known if the APACHE II score, associated with the RIFLE classification, results in greater discriminatory power in relation to mortality in critical patients. Objective. To analyze whether the RIFLE classification adds value to the performance of APACHE II in predicting mortality in critically ill patients. Methods. An observational prospective cohort of 200 patients admitted to the ICU from July 2010 to July 2011 . Results. The age of the sample was $66( \pm 16.7)$ years, $53.3 \%$ female. ICU mortality was $23.5 \%$. The severity of AKI presented higher risk of death: class risk ( $\mathrm{RR}=1.89 \mathrm{CI}: 0.97-3.38, P=0.001)$, grade injury $(\mathrm{RR}=3.7 \mathrm{CI}: 1.71-8.08, P=0.001)$, and class failure $(\mathrm{RR}=$ 4.79 CI:2.10-10.6, $P=0.001$ ). The APACHE II had C-statistics of 0.75, 95\% (CI:0.68-0.80, $P=0.001$ ) and 0.80 (95\% CI:0.74 to $0.86, P=0.001)$ after being incorporated into the RIFLE classification in relation to prediction of death. In the comparison between AUROCs, $P=0.03$. Conclusion. The severity of AKI, defined by the RIFLE classification, was a risk marker for mortality in critically ill patients, and improved the performance of APACHE II in predicting the mortality in this population.
\end{abstract}

\section{Introduction}

The epidemiology, evolution, and treatment of acute kidney injury (AKI) in critically ill patients were better evaluated only after the introduction of Intensive Care Units (ICU) and the introduction of dedicated critical care medicine journals in the 1970s [1]. However, only since the 1980s, scores of disease severity were developed.

Those scores were perfected in the 1990s, highlighting the Acute Physiology and Chronic Health Evaluation (APACHE) [2], the Therapeutic Interventions Scoring System (TISS) [3], and the Sequential Organ Failure Assessment (SOFA) [4]. The APACHE II [5] is the most often cited model in medical literature and the most used nowadays, being recommended by a ministerial order in Brazil since 1998 [6]. These prognostic models are used in the ICU to predict the outcome of patients with certain severe diseases, including acute kidney injury, and the APACHE II score has been the most commonly used predicting instrument in this population [7]. The work done that evaluated the power of APACHE II in predicting the mortality found values ranging from 0.75 to $0.90[6,8,9]$ which were considered excellent. However, the results of the analyses of their performance in subgroups are controversial $[8,10]$ which have stimulated the development of specific models $[11,12]$.

The Acute Dialysis Quality Initiative (ADQI) published, in 2004, the RIFLE classification in an attempt to standardize the definition of acute renal failure [13]. The RIFLE denomination is an acronym which refers to risk (risk of renal dysfunction); injury (injury or damage to the kidney); failure (renal failure); loss (loss of kidney function); end (end stage renal disease) (Table 1).

In several published studies in which the RIFLE classification was used, results showed a linear correlation between the RIFLE score and death, which means that the risk of death increased with the increasing severity of the disease [14-16]. Although the prognostic models have been similar to that shown by the RIFLE classification, to discriminate hospital mortality [17], this classification only takes into account the renal system, among a larger complex of disease severity. 
TABLE 1: RIFLE classification for acute kidney injury.

\begin{tabular}{lll}
\hline Rating & GFR & Urinary output \\
\hline Risk (Risk) & $\uparrow \mathrm{SCr} 1.5 \mathrm{X}$ or $\downarrow$ GFR $>25 \%$ & $<0.5 \mathrm{~mL} / \mathrm{kq} / \mathrm{h}$ for $6 \mathrm{~h}$ \\
Injury (Injury) & $\uparrow \mathrm{SCr} 2 \mathrm{X}$ or $\downarrow \mathrm{GFR}>50 \%$ & $<0.5 \mathrm{~mL} / \mathrm{kq} / \mathrm{h}$ for $12 \mathrm{~h}$ \\
Failure (Failure) & $\uparrow \mathrm{SCr} 3 \mathrm{X}$ or $>4 \mathrm{mg} / \mathrm{dL}$ or $\downarrow \mathrm{GFR}>75 \%$ & $<0.3 \mathrm{~mL} / \mathrm{kq} / \mathrm{h}$ for $24 \mathrm{~h}$ or anuria for $24 \mathrm{~h}$ \\
Function Loss (Loss) & Total loss for 4 weeks + & \\
Final Stage (End) & +3 months for Dialysis & \\
\hline
\end{tabular}

RIFLE: Risk, Injury, Failure, Loss, End; GFR: glomerular filtration rate; SCr: serum creatinine. Adapted [13].

Therefore, it is likely that its performance is not better than the overall scores but may have its discriminatory values added to them.

It is not known if the APACHE II scores, associated with the RIFLE classification, result in greater discriminatory power, in relation to mortality in critically ill patients. Thus, this study was developed to assess whether the RIFLE classification improves the performance of the overall prognostic model of disease severity (APACHE II) in those patients.

\section{Methods}

2.1. Selection of Sample. Inclusion criteria were age older than 18 years and ICU stay longer than 24 hours. The study was conducted in a tertiary hospital in Brazil, from July 2010 to July 2011. We excluded patients with a history of chronic kidney disease or kidney transplant and those who stayed less than 24 hours in the ICU.

All patients, after being informed of the purpose of the study, signed a consent form.

2.2. Study Protocol. This is a prospective observational cohort study, in which patients were followed during their ICU stay until the outcome, discharge, or death. The researcher was not a member of the patient care team and did not participate in therapeutic decisions concerning those individuals. Information about demographics, circumstances which led to hospitalization, and clinical and laboratory data was collected daily from medical records.

The RIFLE classification was used following prerequisites for definition and classification of acute renal failure proposed by the Acute Dialysis Quality Initiative Group [14]. We did not consider the RIFLE evolutionary criteria: loss of renal function and end-stage renal function. The baseline serum creatinine ( $\mathrm{SCr}$ ) was considered as the lowest value found before admission to the ICU. When unknown, baseline serum creatinine was obtained by applying the modification of diet in renal disease (MDRD) [18] simplified formula. We considered as normal a glomerular filtration rate (GFR) of $75 \mathrm{~mL} / \mathrm{min} / 1,73 \mathrm{~m}^{2}$ as follows:

$$
\begin{aligned}
\text { GFR }= & 186 \times-\mid \text { Scr }|\times 1.154 \times-| \text { age } \mid \\
& \times 0.203 \mid 0.742 \text { if female }|\times| 1.210 \text { if black } \mid .
\end{aligned}
$$

The criterion for measuring urine flow was adapted. Although all patients were using indwelling vesical catheters, allowing continuous measure and hourly recording of urine flow, only the cumulative volume was assessed within 24 hours. The weight of the patients was estimated at $60 \mathrm{~kg}$ assuming an average weight of well-nourished adults. Patients were classified into three categories: risk (urine flow $<30 \mathrm{~mL} / \mathrm{h}$ ), injury (urine flow $<18 \mathrm{~mL} / \mathrm{h}$ ), and, Failure (urine flow $<4 \mathrm{~mL} / \mathrm{h}$ ). Analyses of the criteria for diagnosis and classification of acute kidney injury were later conducted. The outcome of interest was ICU mortality.

We defined 24-hour RIFLE as the group of patients with acute kidney injury in the first 24 hours after ICU admission and RIFLE-1 as the group of patients with acute kidney injury at any time during their ICU stay.

For definition of sepsis and septic shock the 1991 Consensus Conference criteria settings were used [19].

The APACHE II [5] was calculated keeping the score for renal dysfunction, not to overestimate the RIFLE increment predictor. To avoid time-dependent bias, APACHE II was evaluated within 24 hours of admission and on the RIFLE1 day. The most abnormal values of vital signs and laboratory exams were used. TISS scores 28 [3] and the Glasgow Coma Scale [20] (GCS) were calculated only on admission. In sedated patients GCS was recorded as the state of consciousness measured immediately before sedation. We collected all the data needed for the calculation of prognostic scores.

\section{Statistical Analysis}

Statistical analyses were performed using SPSS version 17.0 Software (SPSS, Inc., Chicago, IL, USA). Descriptive statistics were used to characterize the population.

Continuous variables were presented as mean \pm standard deviation or as median and interquartile range, according to distribution. Categorical variables were analyzed using the $X^{2}$ test or Fisher's exact test.

Logistic regression analysis was used to evaluate the impact of the RIFLE classification on the occurrence of mortality, adjusted for the prognosis model in question (APACHE II).

The predictive ability of the proposed prognostic model (APACHE II score and APACHE II score incorporated into RIFLE) was assessed by the area under the curve [21] (ROC) receiver operator characteristic. To compare the ROC curves the MedCalc Software Version 12.3.0.0, Mariakerke, Belgium was used. Calibration model was performed using the Hosmer-Lemeshow [22]. 
TABLE 2: Demographic and clinical characteristics of critically ill patients defined by the RIFLE classification.

\begin{tabular}{|c|c|}
\hline Variable & $N=200$ \\
\hline Age (years) $( \pm \mathrm{DP})$ & $66( \pm 16.7)$ \\
\hline Females $N(\%)$ & $107(53.5)$ \\
\hline Days of ICU stay (IQR) & $12(4-17)$ \\
\hline \multicolumn{2}{|l|}{ Comorbidities on admission $N(\%)$} \\
\hline $\mathrm{DM}+\mathrm{SH}$ or DLP & $99(49.5)$ \\
\hline Cancer/Oncology Therapy & $34(17)$ \\
\hline NYHA class IV & $06(3)$ \\
\hline Immunossuppression & $3(1.5)$ \\
\hline \multicolumn{2}{|l|}{ Surgical Admission N (\%) } \\
\hline ICU Admission & $65(32.5)$ \\
\hline \multicolumn{2}{|l|}{ Compromised system $N(\%)$} \\
\hline Respiratory & $54(27.3)$ \\
\hline Neurology & $52(26.3)$ \\
\hline Heart & $43(21.7)$ \\
\hline Polytrauma & $02(1)$ \\
\hline Other & $47(23)$ \\
\hline Use of Mechanical Ventilation N (\%) & $79(39.5)$ \\
\hline Vasoactive drugs $N(\%)$ & $55(27.5)$ \\
\hline Diuretic use $N(\%)$ & $54(27)$ \\
\hline Mean arterial pressure (SD) & $94( \pm 26.7)$ \\
\hline Serum creatinine (minimum-maximum) & $1(0.2-9.8)$ \\
\hline APACHE II score $( \pm S D)$ & $13( \pm 6.6)$ \\
\hline Not patched APCHE II renal $( \pm S D)$ & $12.3( \pm 5.9)$ \\
\hline SOFA (IQR) & $3(0-5)$ \\
\hline Nonrenal SOFA (IQR) & $2(0-4)$ \\
\hline TISS-28 ( \pm SD $)$ & $21( \pm 7.3)$ \\
\hline Glasgow $( \pm \mathrm{SD})$ & $13.3( \pm 3)$ \\
\hline
\end{tabular}

RIFLE: Risk, Injury, Failure, Loss, End: DM: Diabetes Mellitus; Hypertension: hypertension DLP: dyslipidemia; NYHA class IV: heart failure functional class IV; SOFA: Sequential Organ Failure Assessment Score; Nonrenal SOFA: Sequential Organ Failure Assessment Score without the score for renal failure. APACHE II: Acute Physiology and Chronic Health Evaluation version II. Nonrenal APACHE II: APACHE II score without referring to kidney failure. TISS-28: The Therapeutic Intervention Score System; Glasgow: Glasgow Coma Scale; SD: standard deviation; IQR: Interquartile range.

\section{Results}

The study sample consisted of 200 patients, of whom 53\% were female, and mean age was 66 years $( \pm 16.7)$. Nonsurgical admissions were more frequent than surgical admissions (67.5\% versus $32.5 \%)$, 27\% with impaired respiratory tract, followed by $26 \%$ with neurological injuries and $22 \%$ due to cardiac causes. The length of ICU stay was 12 (IQR: 4-17) days. The value of the APACHE II score was $13.3( \pm 6.6)$ and $12.3( \pm 5.9)$ when scores equivalent to renal dysfunction was withdrawn. The values of TISS-28 score were $21( \pm 7.3)$ (Table 2).

The frequency of acute kidney injury within 24 hours of ICU stay was $42 \%$ classified as class risk $7 \%$, class injury $15 \%$, and class failure $19 \%$. On the day of discharge or death the percentage was $47.5 \%$. The causes most often associated with

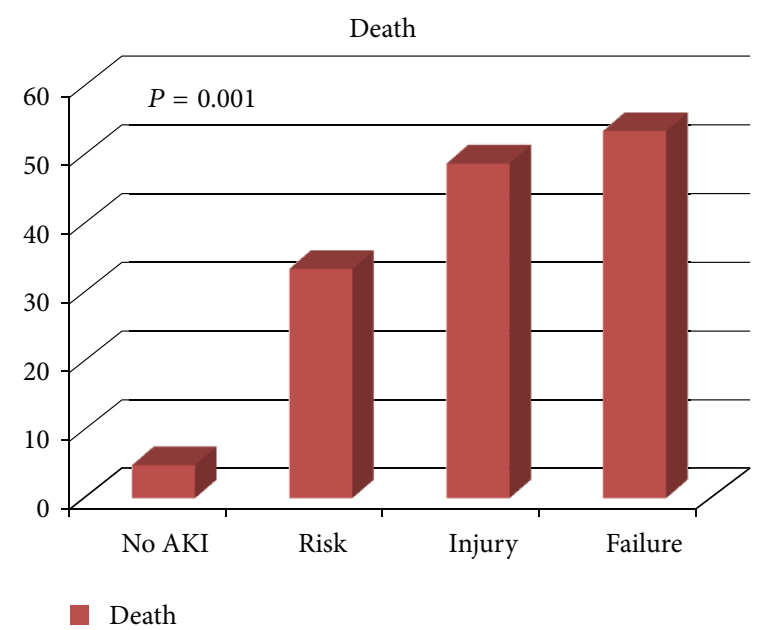

FIGURE 1: Frequency of mortality according to the RIFLE classification.

TABLE 3: The Impact of the RIFLE classification criterion in ICU mortality, adjusted for APACHE II score.

\begin{tabular}{lcccc}
\hline Variable & OR & df & IC 95\% & $P$ value \\
\hline APACHE II & 1.66 & 1 & $1.22-2.27$ & 0.001 \\
RIFLE 24 h & 1.07 & 1 & $1.00-1.13$ & 0.026 \\
APACHE II in RIFLE-1 & 1.04 & 1 & $0.98-1.10$ & 0.168 \\
RIFLE-1 & 2.17 & 1 & $1.50-3.14$ & 0.001 \\
\hline
\end{tabular}

OR: Oddis Ration (Odd Ratio); df: degrees of freedom; CI: Confidence Interval; APACHE II: Acute Physiology and Chronic Health Evaluation version II; RIFLE $24 \mathrm{~h}$ : RIFLE-Risk, Injury, Failure, Loss, End defined in 24 hours of ICU stay; RIFLE-1 Risk, Injury, Failure, Loss, End set on the day of AKI during their ICU stay.

the development of acute kidney injury were septic shock $47 \%$, sepsis $23 \%$, and low cardiac outputs $17 \%$ and $13 \%$ of other causes. Overall mortality was $25.5 \%$.

The progressive severity of AKI according to RIFLE criteria for subgroups risk, injury, and failure was associated with increased mortality when compared to patients without AKI (Figure 1). The risk of death was thus rated: class risk (RR $=1.8995 \% \mathrm{CI}: 0.97$ to $3.38, P=0.001)$; class injury $(\mathrm{RR}=3.7$ 95\% CI: $1.71-8.08, P=0.001)$; class failure $(\mathrm{RR}=4.7995 \% \mathrm{CI}$ 2.10 to $10.6, P=0.001)$.

The RIFLE classification was associated with mortality independent of APACHE II score (Table 3).

It was observed that the APACHE II score calculated within 24 hours after admission to the ICU showed $C$ statistics of 0.73 (95\% CI: 0.66 to $0.81, P=0.001$ ) in relation to prediction of death. After incorporating the RIFLE score, the APACHE II score showed statistically C-0.77 (95\% CI: 0.70 to $0.84, P=0.001)$. The APACHE II when calculated on the day of renal injury at any time during their ICU stay had $C$ statistics of 0.75 (95\% CI: 0.68 to $0.80, P=0.001)$ in relation to prediction of death, and after incorporating RIFLE 1 score, the APACHE II had C-statistics of 0.80 (95\% CI: 0.74-0.86, $P=0.001$ ) (Table 4).

Comparing the ROC curves, there was no significant difference between RIFLE and APACHE II associated with 
TABLE 4: Comparative analysis of discrimination and calibration of APACHE II in predicting mortality in the ICU alone and when combined with the RIFLE score calculated within 24 hours after ICU admission and on the Day of AKI.

\begin{tabular}{lcccc}
\hline \multirow{2}{*}{ Score } & \multicolumn{2}{c}{ Discrimination } & \multicolumn{2}{c}{ Calibration } \\
& Area under the ROC curve & CI 95\% & $P P$ & GOF \\
\hline APACHE II 24h & 0.74 & $0.66-0.81$ & 0.001 & 9.6 \\
APACHE II + RIFLE 24h & 0.77 & $0.70-0.84$ & 0.001 & 0.289 \\
APACHE II in RIFLE-1 & 0.75 & $0.68-0.80$ & 0.001 & \multirow{2}{*}{0.12} \\
APACHE II + RIFLE-1 & 0.80 & $0.74-0.86$ & 0.001 & \\
\hline
\end{tabular}

ROC: receiver operating characteristic; IC: confidence interval; GOF: goodness of fit; APACHE II: Acute Physiology and Chronic Health Evaluation version II; RIFLE 24 h: RIFLE-Risk, Injury, Failure, Loss, End defined within 24 hours of ICU stay; RIFLE-1 Risk, Injury, Failure, Loss, End set on the day of AKI during ICU stay.

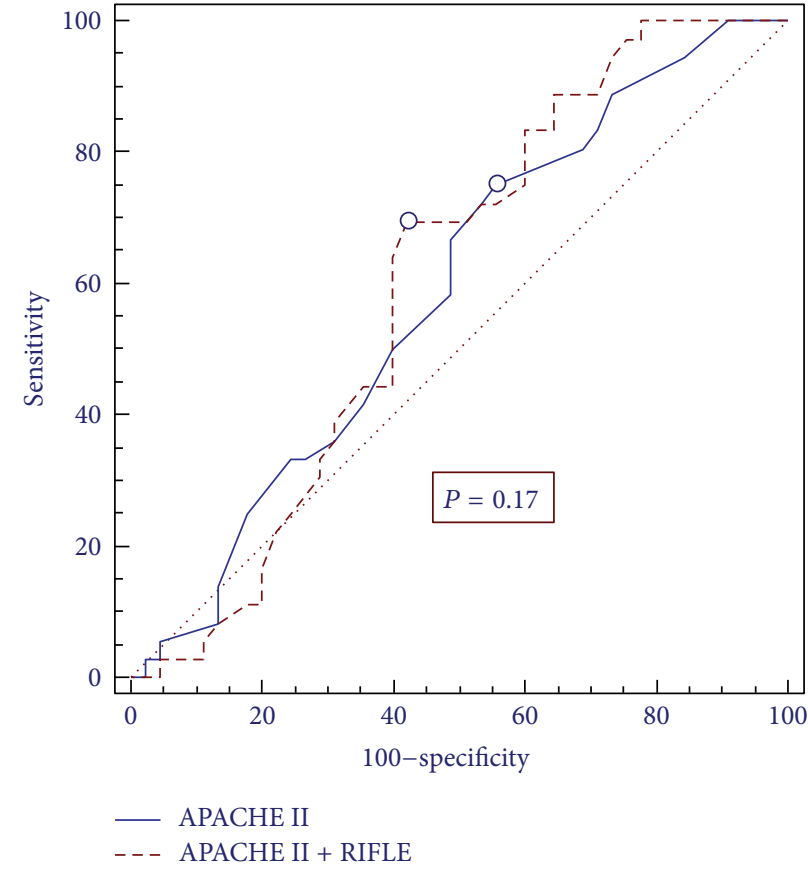

(a)

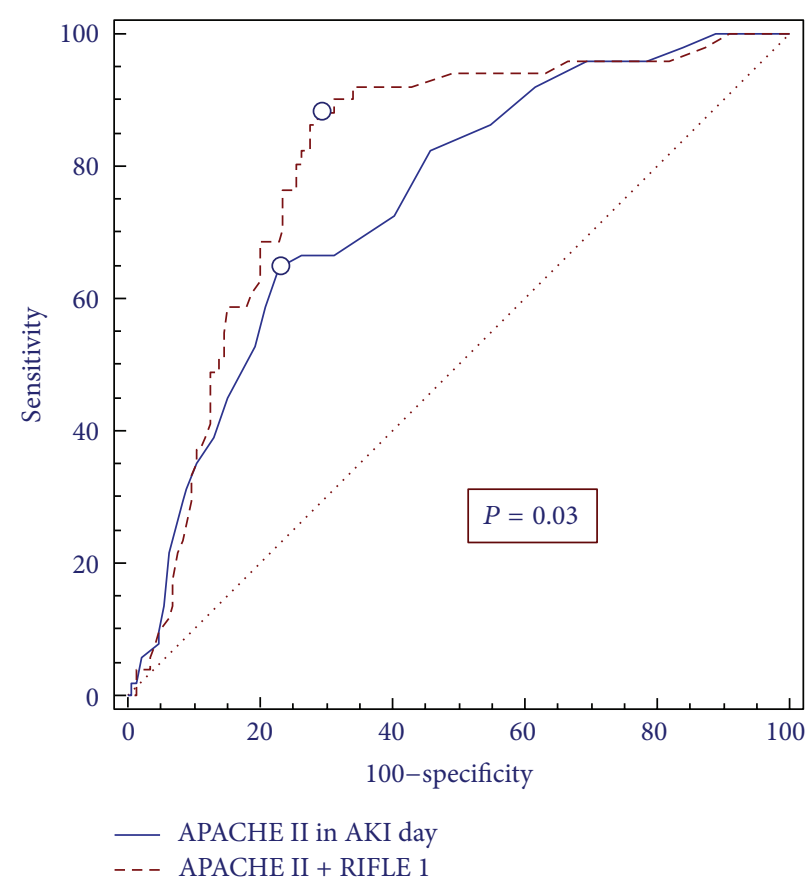

(b)

FIGURE 2: Comparative analysis between the ROC curves and the APACHE II score and calculated after incorporating the RIFLE score. (a) Defined in 24 hours after ICU admission. (b) defined at any time during ICU stay.

RIFLE, $P=0.17$, when both set within 24 hours of admission, but there was significant difference between the RIFLE and Apache II associated with RIFLE, $P=0.03$ when both were defined on the day of AKI (Figure 2).

\section{Discussion}

The findings of this study have demonstrated that the RIFLE score added value to the performance of APACHE II in predicting mortality. A likely explanation is that the criterion of reduction in urine flow set $73 \%$ of the cases of AKI in the most severe RIFLE score, failure. The class failure was independently associated with mortality thus by incorporating the RIFLE classification, the APACHE II included a clinical feature of acute kidney injury associated with shorter survival: oliguria.
It was also observed that the APACHE II score showed discriminatory power in relation to ICU mortality and similarly when evaluated within 24 hours and on the day of the AKI. Conceptually, the APACHE II includes 12 physiologic variables recorded at their worst values within the first 24 hours of hospitalization. One approach that includes physiological variables without time limitation would be more accurate to predict survival chance like, for example, the value of serum creatinine, than an approach that considers those variables only during admission.

Previous studies that evaluated the performance of APACHE II demonstrated that a prognostic model developed from the general population of patients can have controversial performance in specific subgroups, such as patients with AKI. The authors who found unsatisfactory performance of APACHE II score in discriminating mortality when compared to other prognostic models attributed such 
performance to timede-pendent bias, as in the study of Maccariello et al. [7]. Those authors evaluated the performance of six prognostic models in critically ill patients and the need for dialysis and found that discrimination was bad for all models. The authors calculated the scores in the 24 hours after ICU admission.

On the other hand, Fernandes et al. [8] compared the performance of the APACHE II score with a specific score, the ATN-ISS [11] (individual severity scores of acute tubular necrosis) in patients admitted within and outside the ICU, calculated on the day of assessment by a nephrologist, and found that in the group admitted to the ICU the APACHE II score presented $C$-statistics of 0.75 , a result similar to that found in the present study. The study by Parker et al. [23] (1998) demonstrated that the best time to risk stratify patients with AKI is the use of APACHE II score on the day of dialysis, particularly when modified for the presence or absence of urinary flow (C-statistics: 0.74 versus $0.80, P=0.005)$, but in that study a comparison, at different times, was not carried out.

This study demonstrated that APACHE II score did not improve its performance significantly when incorporated into the RIFLE classification, defined in the 24 hours after ICU admission. This finding could be associated with higher mortality in the group that developed AKI during their ICU stay, compared to patients who developed AKI within 24 hours of admission (67\% versus 22\%). All the patients who recovered renal function, partially or totally, had developed AKI within 24 hours of admission, while the cases of developed AKI during ICU stay progressed to more severe classes. This finding confirms the idea that the development of AKI in critically ill patients is associated with disease severity.

However, some considerations must be made: first, when the value of baseline serum creatinine is unknown, the ADQI recommends estimating its value calculated by the formula of MDRD [18], but published results on its accuracy are conflicting. Although only $20 \%$ of baseline SCr has been calculated in this study, this may have contributed to misclassification. It is known that calculated serum creatinine does not replace real creatinine, but validation of the MDRD was not the objective of this study, and second, patients are evaluated from a single research center, which requires caution when extrapolating the data presented here for other services.

A highlight of this study is to be the first to perform a simultaneous evaluation of a general prognostic score of disease severity, APACHE II score, associated with a specific one the RIFLE score. Moreover, there are few studies that have evaluated the RIFLE score in a prospective analysis, complying with the standards recommended by ADQI. A "real-time" analysis approaches the daily reality of intensive care and provides more reliability to the data collected and statistical analyses.

The results of this study indicate that the RIFLE classification helped to improve the performance of APACHE II in predicting mortality in critically ill patients, when set at any time during the patient's stay in ICU. They also demonstrated that the severity of kidney injury is associated with disease severity and mortality in this population. It is noteworthy that although the scores help in discussions about prognosis and risk stratification, no single model should be used to define treatments or conduct.

\section{Conflict of Interests}

Each of the authors declares no financial or other conflict of interests in relationship with a company/organization or some commercial identities such as "SPSS, Inc. Chicago, IL, USA, and MedCalc Software Version 12.3.0.0" that could benefit financially from the publication of the data in their paper.

\section{References}

[1] R. Bellomo, "The epidemiology of acute renal failure: 1975 versus 2005," Current Opinion in Critical Care, vol. 12, no. 6, pp. 557-560, 2006

[2] W. A. Knaus, J. E. Zimmerman, D. P. Wagner, E. A. Draper, and D. E. Lawrence, "APACHE-acute physiology and chronic health evaluation: a physiologically based classification system," Critical Care Medicine, vol. 9, no. 8, pp. 591-597, 1981.

[3] D. R. Miranda, A. De Rijk, and W. Schaufeli, "Simplified therapeutic intervention scoring system: the TISS-28 itemsresults from a multicenter study," Critical Care Medicine, vol. 24, no. 1, pp. 64-73, 1996.

[4] J.-L. Vincent, R. Moreno, J. Takala et al., "The SOFA (Sepsisrelated Organ Failure Assessment) score to describe organ dysfunction/failure," Intensive Care Medicine, vol. 22, no. 7, pp. 707-710, 1996.

[5] W. A. Knaus, E. A. Draper, D. P. Wagner, and J. E. Zimmerman, "APACHE II: a severity of disease classification system," Critical Care Medicine, vol. 13, no. 10, pp. 818-829, 1985.

[6] J. R. Rocco, M. Soares, P. F. Cariello et al., "Perfomace of eight prognostic scores in patients admitted to the intensive care unit," Revista Brasileira de Terapia Intensiva, vol. 17, pp. 165-169, 2005.

[7] E. Maccariello, C. Valente, L. Nogueira et al., "Performance of six prognostic scores in critically Ill patients receiving renal replacement therapy," Revista Brasileira de Terapia Intensiva, vol. 20, pp. 115-123, 2008.

[8] N. M. D. S. Fernandes, P. A. D. S. Pinto, T. B. D. P. Lacet et al., "APACHE II and ATN-ISS in acute renal failure (ARF) in intensive care unit (ICU) and non-ICU," Revista da Associacao Medica Brasileira, vol. 55, no. 4, pp. 434-441, 2009.

[9] R. C. Sachdeva, "Statistical basis and clinical applications of severity of illness scoring systems in the intensive care unit," Current Opinion in Critical Care, vol. 5, no. 3, pp. 180-183, 1999.

[10] A. L. Balbi, D. P. Gabriel, R. C. Barsante, J. T. Caramori, L. C. Martin, and P. Barreti, "Assessment of mortality and specific index in acute renal failure," Revista da Associacao Medica Brasileira, vol. 51, no. 6, pp. 318-322, 2005.

[11] F. Liaño, A. Gallego, J. Pascual et al., "Prognosis of acute tubular necrosis: an extended prospectively contrasted study," Nephron, vol. 63, no. 1, pp. 21-31, 1993.

[12] R. L. Mehta, M. T. Pascual, C. G. Gruta, S. Zhuang, and G. M. Chertow, "Refining predictive models in critically ill patients with acute renal failure," Journal of the American Society of Nephrology, vol. 13, no. 5, pp. 1350-1357, 2002. 
[13] R. Bellomo, C. Ronco, J. A. Kellum, R. L. Mehta, and P. Palevsky, "Acute renal failure- definition, outcome measures, animal models,fluid therapy and information technology needs," Critical Care, vol. 8, no. 4, pp. R204-R212, 2004.

[14] M. Ostermann and R. W. S. Chang, "Acute kidney injury in the intensive care unit according to RIFLE," Critical Care Medicine, vol. 35, no. 8, pp. 1837-1843, 2007.

[15] P. Piccinni, D. N. Cruz, S. Gramaticopolo et al., "Prospective multicenter study on epidemiology of acute kidney injury in the ICU: a critical care nephrology Italian collaborative efort (NEFROINT)," Minerva Anestesiologica, vol. 77, no. 11, pp. 10721083, 2011.

[16] Z. Ricci, D. Cruz, and C. Ronco, "The RIFLE criteria and mortality in acute kidney injury: a systematic review," Kidney International, vol. 73, no. 5, pp. 538-546, 2008.

[17] S. Uchino, J. A. Kellum, R. Bellomo et al., "Acute renal failure in critically ill patients: a multinational, multicenter study," Journal of the American Medical Association, vol. 294, no. 7, pp. 813-818, 2005.

[18] A. S. Levey, J. P. Bosch, J. B. Lewis, T. Greene, N. Rogers, and D. Roth, "A more accurate method to estimate glomerular filtration rate from serum creatinine: a new prediction equation," Annals of Internal Medicine, vol. 130, no. 6, pp. 461-470, 1999.

[19] R. C. Bone, R. A. Balk, F. B. Cerra et al., "Definitions for sepse and organ failure and guidelines for the use of innovative therapies in sepse. The ACCP/SCCM Consensus Conference. American College of chest physicians/Society of Critical Care Medicine Consensus Conference Committee," Chest, vol. 101, pp. 1644-1655, 1992.

[20] G. Teasdale and B. Jannett, "Assessment of coma and empaired consciusness," Lancet, vol. 13, pp. 81-83, 1974.

[21] J. A. Hanley and B. J. McNeil, "The meaning and use of the area under a receiver operating characteristic (ROC) curve," Radiology, vol. 143, no. 1, pp. 29-36, 1982.

[22] S. Lemeshow and D. W. Hosmer Jr., "A review of goodness of fit statistics for use in the development of logistic regression models," American Journal of Epidemiology, vol. 115, no. 1, pp. 92-106, 1982.

[23] R. A. Parker, J. Himmelfarb, N. Tolkoff-Rubin, P. Chandran, R. L. Wingard, and R. M. Hakim, "Prognosis of patients with acute renal failure requiring dialysis: results of a multicenter study," American Journal of Kidney Diseases, vol. 32, no. 3, pp. 432-443, 1998. 


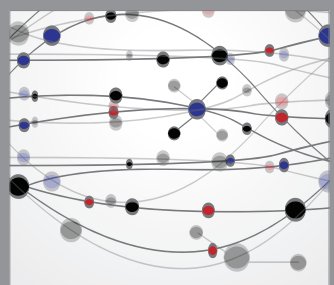

The Scientific World Journal
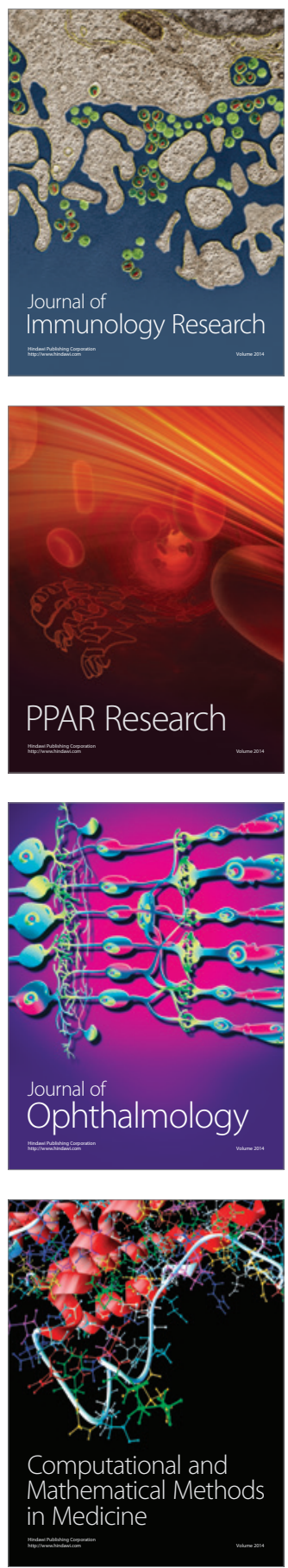

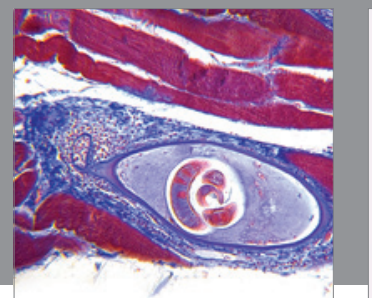

Gastroenterology

Research and Practice
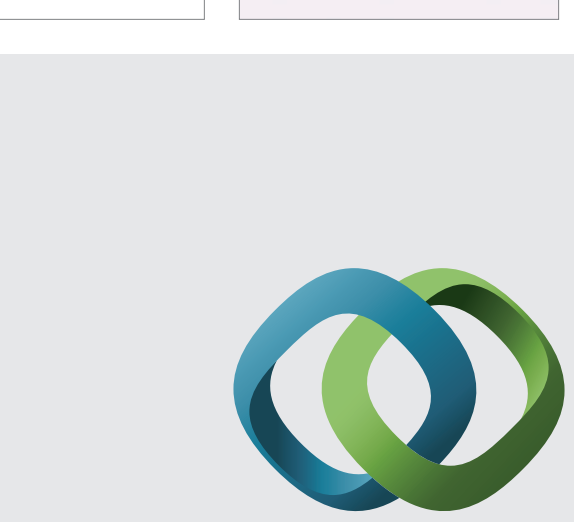

\section{Hindawi}

Submit your manuscripts at

http://www.hindawi.com
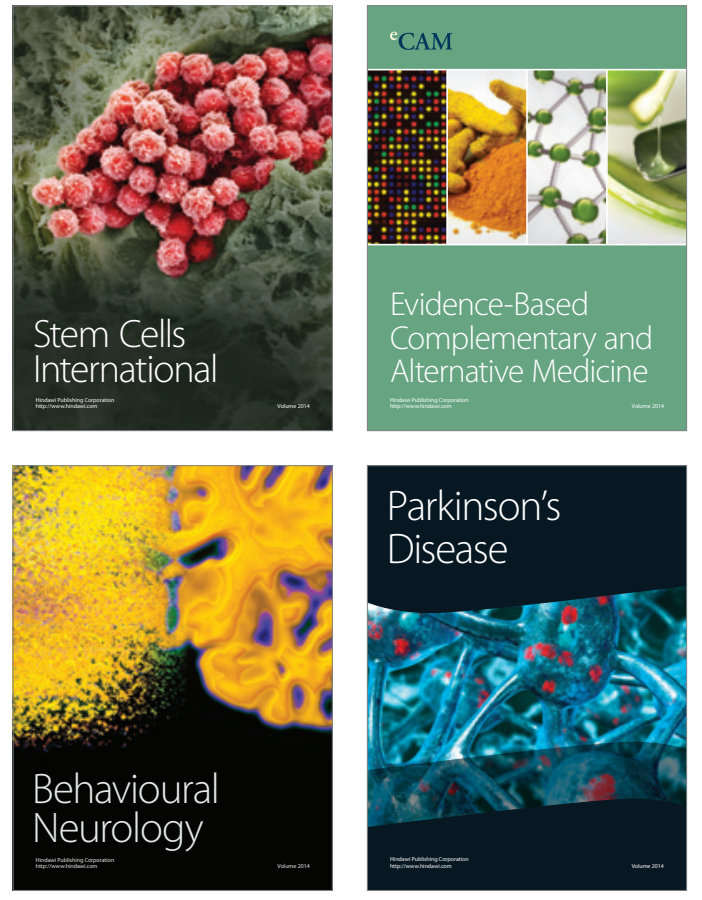
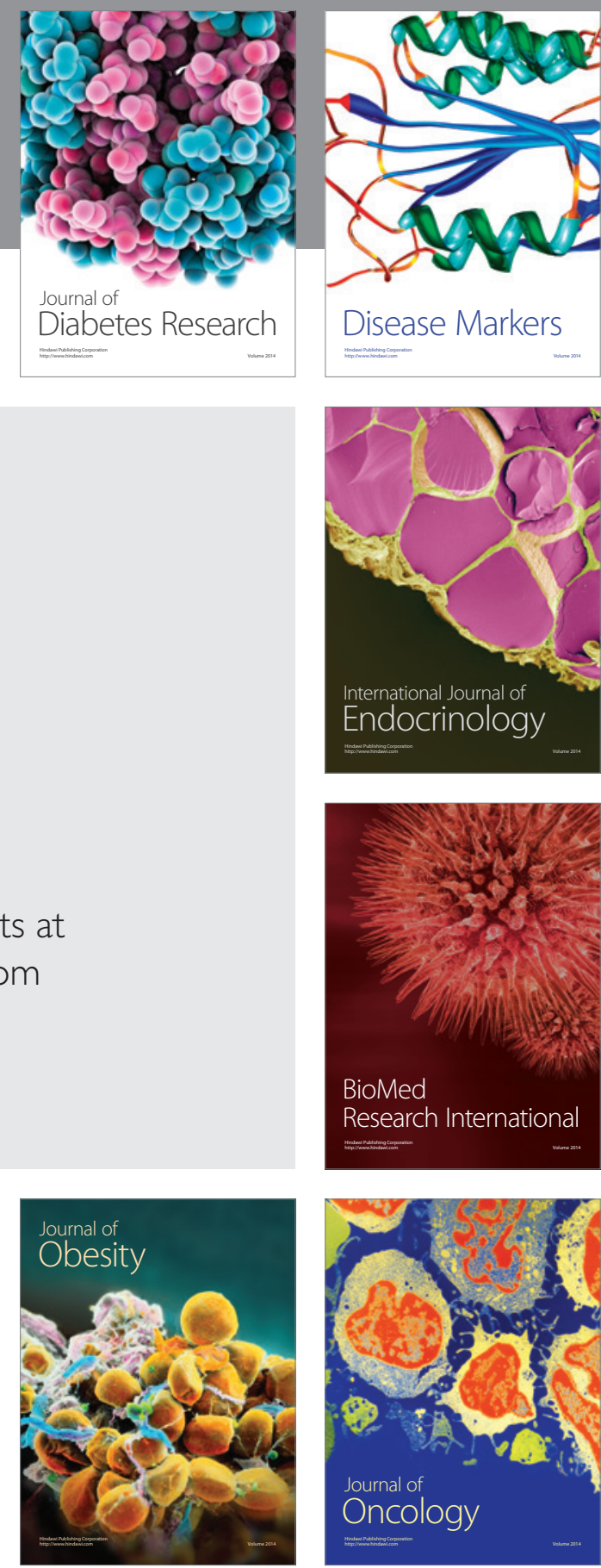

Disease Markers
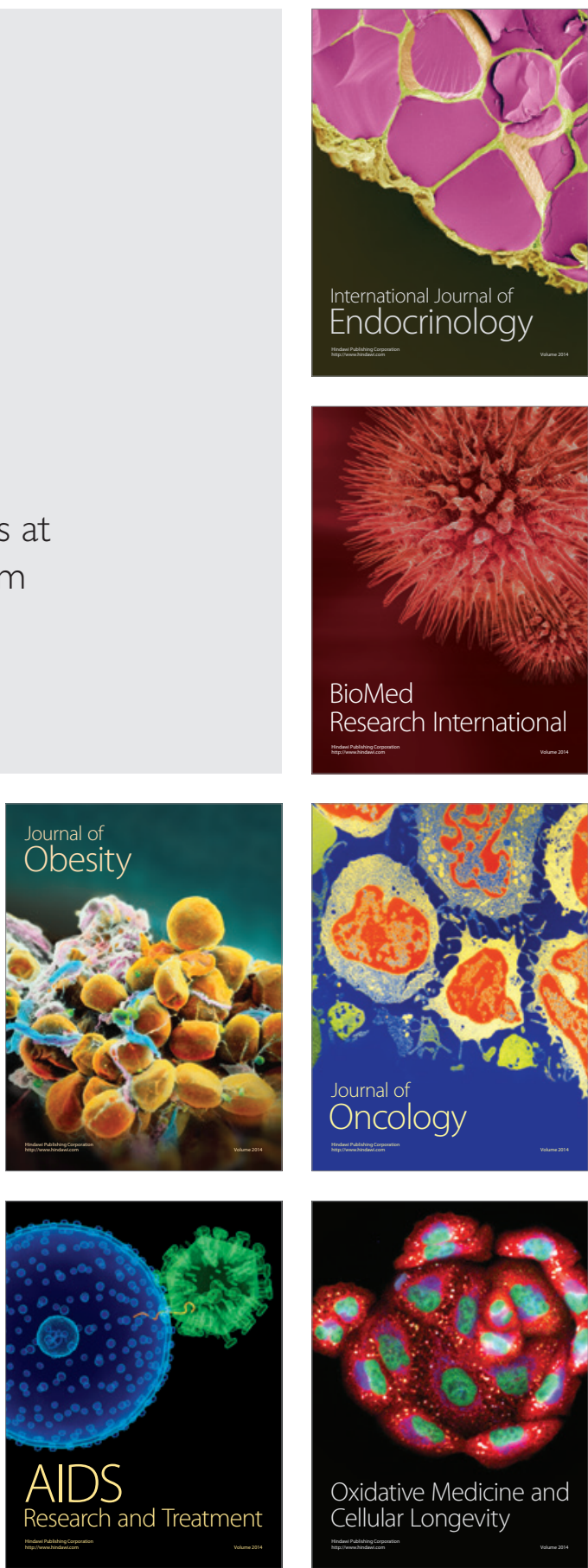